undermine its effectiveness.

A number of commentators point out the irony that many of the hotly debated flexible mechanisms are there at the insistence of the United States, which is highly unlikely to ratify the Kyoto Protocol.

The November meeting coincides with the US elections, and the US position is not expected to have changed by then. However, the United States does not have a blocking vote, so the protocol can be implemented without it. The new administration could still instigate mitigation measures if it chose to do so.

But observers point out that Kyoto's $5.2 \%$ target would not be reached without US involvement. Benito Muller, a senior research fellow from the Oxford Institute for Energy Studies, says that although this is "neither here nor there", more substantial steps are needed. "Industrialized nations have committed themselves to showing leadership [on global warming] and Kyoto is the tool by which we can do it," he says.

A continuing source of controversy involves a mechanism that would allow industrialized nations to claim credits against their emissions by investing in clean technologies - such as nuclear power - in developing countries.

The nuclear industry is emphasizing the major role that nuclear energy could play in reducing global warming. But critics, including Britain's Royal Commission, warn that the issue of nuclear waste must be dealt with before it can qualify as a clean technology. http://www.rcep.org.uk/newenergy.html

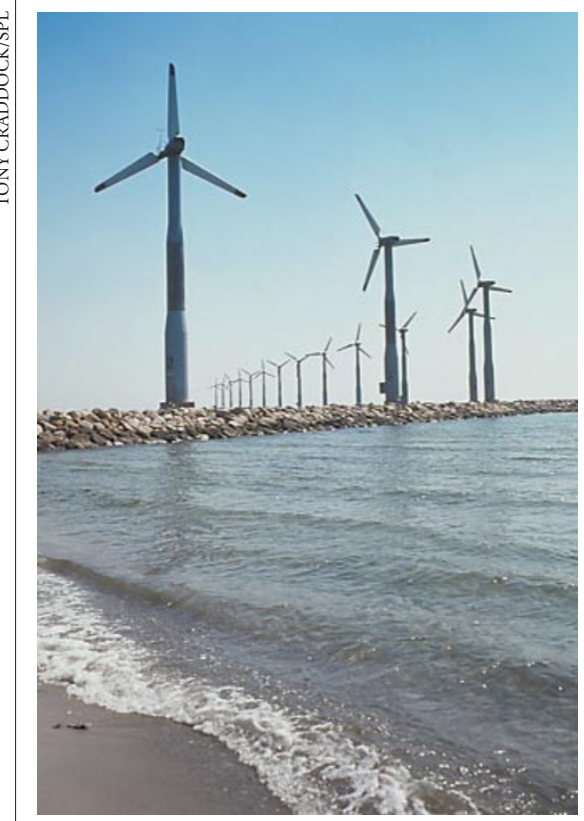

Clean power: wind turbines, such as these in Jutland, Denmark, are a rare sight in Britain.

\section{Brookhaven collider opens its quest for Big Bang conditions}

Steve Nadis, Boston

Two gold nuclei, hurtling in opposite directions around a 2.4-mile ring, crashed into each other last week, unleashing $10 \times 10^{12}$ electron volts of energy. This startling event marked the debut of the Relativistic Heavy Ion Collider (RHIC), as it came online at Brookhaven National Laboratory in New York.

Over the next few weeks, scientists hope to increase the accelerator's power to generate collisions releasing about $40 \times 10^{12}$ electron volts of energy. The aim is to simulate the conditions that existed during the first few microseconds after the Big Bang.

"RHIC can produce the largest centre of mass energy achieved by any accelerator, which makes it the best machine for studying the birth of nuclear matter in the Universe," says Tom Kirk, Brookhaven's associate director for high-energy and nuclear physics.

Researchers at the collider are trying to create a plasma of unattached quarks and gluons - the state of matter thought to have existed just after the Big Bang. They want to study the 'phase transition' that occurs as these particles combine to form protons and neutrons.

In February, scientists using the Super Proton Synchrotron (SPS) at the European Laboratory for Particle Physics (CERN) in Geneva announced they had found "compelling evidence" for the quark-gluon plasma (see Nature 403, 581; 2000). But the SPS did not generate enough energy for researchers to see the plasma directly.

The RHIC, which is about 10 times more powerful, "should produce the energy unequivocally needed to see the plasma”, says Kirk. The collider will yield temperatures well above one trillion degrees kelvin at the site of collision.

RHIC scientists will scour the rubble left by the ion collisions for clues to the plasma's existence. One tell-tale sign would be gamma-ray radiation produced by interactions of unconfined, high-energy quarks in the plasma. "That would certainly be a smoking gun," says Brookhaven physicist Tom Ludlam. Other direct evidence of the plasma's existence could come from electrons emerging from the plasma before it cools and reverts to ordinary nuclear matter. This cooling is very quick -
Collider scope? An end view of a collision of two gold nuclei at Brookhaven's RHIC collider.

the plasma is expected to last about $10^{-23}$ seconds.

The researchers also hope to find out the precise value of the critical temperature at which the quarks in protons and neutrons become unconfined - or, conversely, the into nucleons. And by measuring specific properties of the quark-gluon plasma, such as its temperature, density and entropy, they plan to test key predictions of quantum chromodynamics, the theory describing the strong nuclear force that joins quarks with gluons.

The RHIC machine was switched on after a concerted effort by Brookhaven officials to allay public fears of doomsday scenarios stirred up by alarmist news coverage. These media accounts suggested that the dense plasma created in the RHIC's high-energy collisions could produce a miniature black hole or a previously unseen form of nuclear matter called 'strange matter' — either of which might, according to the scare stories, quickly devour the planet. A panel of physicists dismissed these concerns in a report issued last autumn.

The current experiment at the RHIC is scheduled to run until August. The machine will eventually be superseded by the 30times more powerful A Large Ion Collider Experiment (ALICE) at CERN's Large Hadron Collider, which is due to be started up in 2005. "But there is a lot to learn before ALICE is turned on,” says Ludlam. "This really is unexplored territory." temperature at which free quarks coalesce 